\title{
Accelerated Life Tests Electrochemical Accumulators
}

\author{
K. V. Bezruchko*, A. O. Davidov, S. V. Shirinsky \\ Laboratory of autonomous energetics, National aerospace university, Kharkov, 61070, Ukraine
}

\begin{abstract}
The theoretical basis of the method of accelerated life tests of nickel-cadmium batteries have been considered. The effect of self-discharge of a resource of nickel-cadmium batteries running in standby mode is shown. The effect of temperature on the rate of self-discharge $\mathrm{Ni}-\mathrm{Cd}$ batteries is shown.
\end{abstract}

Keywords Nickel-cadmium battery, resource, accelerated testing

\section{Introduction}

Chemical current sources are an integral part of the energy systems of autonomous objects. In power systems with lowlife or low power can be used by primary chemical current sources. However, with increasing service life and the power of autonomous objects, the application of primary power sources have become impractical. In this case the electrochemical batteries are used. At the moment in power supply systems of autonomous objects, especially objects aerospace engineering, the most nickel-cadmium batteries are widely used.

Lifespan of power plants of autonomous objects largely depends on the characteristics of electrochemical batteries. Therefore, the definition of the resource electrochemical batteries is crucial in determining the service life characteristics of space-rocket complexes. Battery life is due to the nature of the flow rate and degradation processes. The speed and nature of these processes depend strongly on the mode and conditions of use.

Modern techniques of electrochemical battery life tests have significant drawbacks:

1. techniques associated with direct life tests in real time. As a rule they are carried for a long time. So by the time the test results the actuality results is substantially reduced relevance due to the emergence of new types of batteries;

2. techniques based on statistical data processing. It is necessary the preliminary testing in real time. Do not allow confirmation of the fact that the mechanism of degradation has not changed under the influence of stress factors. They have low reliability of the results. It is also not always appropriate and is not always possible to obtain statistical material for large quantities of accumulators;

3. methods of accelerated life tests of batteries. These methods are based on studding of the physical nature of

* Corresponding author:

autonomenergy@khai.edu (K.V. Bezruchko)

Published online at http://journal.sapub.org/eee

Copyright (C 2012 Scientific \& Academic Publishing. All Rights Reserved degradation processes. Despite the obvious advantages, they practically not used due to lower accuracy and reliability of the results due to the impact of unaccounted factors.

\section{The Developing Method of Acceler- ated Life Tests of Electrochemical Batteries}

In the present perspective is the development of techniques based on the studding of the physical nature of degradation processes. These methods allow to solve the problem of determining the resource battery with a lack of statistical data. For the successful application of such techniques requires careful studding of the mechanisms of degradation and the factors influencing the nature and intensity of these processes.

During normal operation the battery is affected by many external factors. These impacts will inevitably affect the internal state of the battery. Changing the internal state of batteries changed the nature and intensity of the effect of various factors on the internal characteristics of the cells (Figure 1). Typically, this effect is negative and leads to poor performance, i.e. to their degradation (Figure 2).

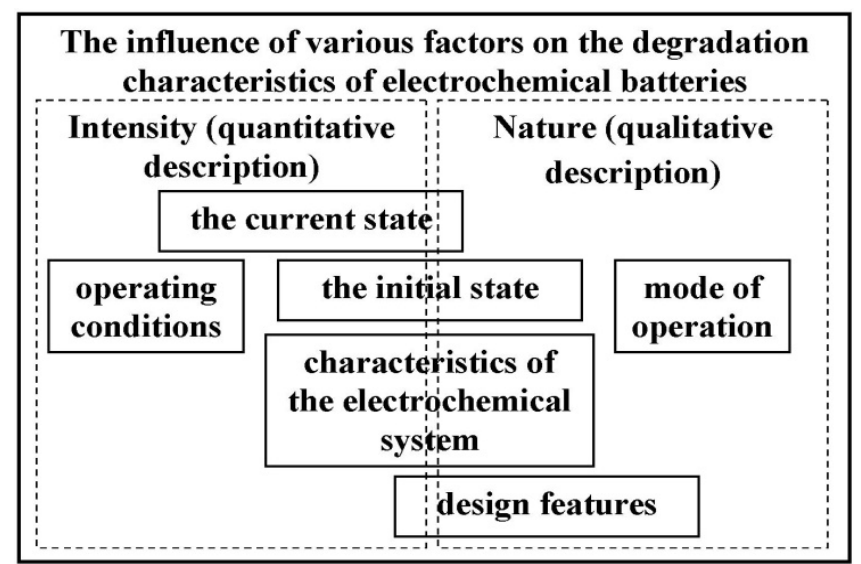

Figure 1. The factors which are determeddetermining the nature and intensity of degradation processes 
It should be noted that the nature of degradation processes (a set of the most common effects of degradation for a given size battery) is primarily depended on the mode of operation of these batteries.[1,4,5] At the same time, the intensity of degradation processes is determined by environmental conditions and the current state of the battery.

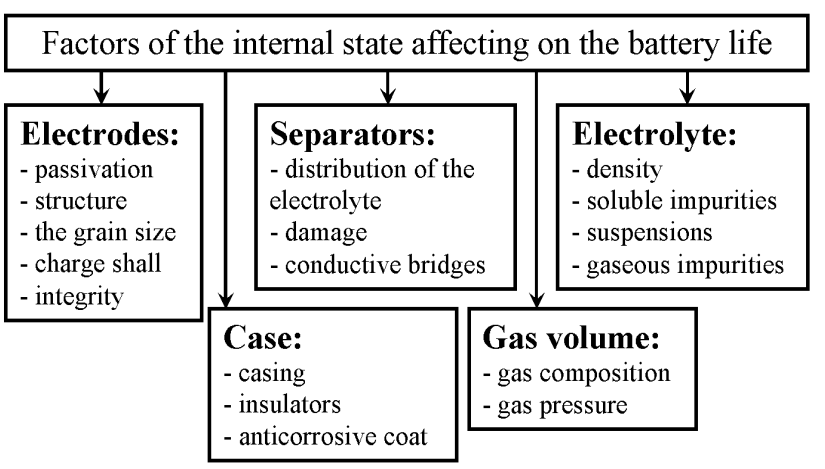

Figure 2. Factors internal state, affecting the rate of change of the resource

Batteries power systems of space-rocket complexes, depending on the specifics of their operation, may be exposed to various external factors, the most important of which are shown in Figure 3.

\section{Forcing factors on the battery during operation}

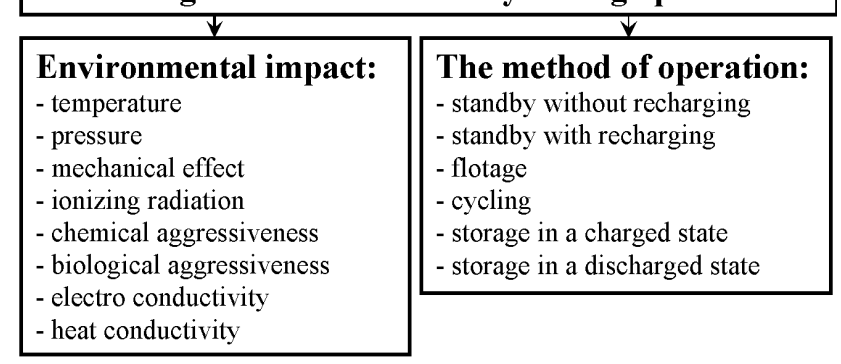

Figure 3. Forcing factors affecting the rate of change of battery resource

Typically, simulations of different states of the environment is used in the climate and accelerated environmental testing to study the characteristics of the battery under these conditions. In the accelerated life tests is more important to study the influence of individual factors on the degradation of the battery to control the speed of degradation.

It is known that the greatest influence on the electrochemical system has a temperature. Optimal in terms of resource exploitation is the battery in a temperature range of 5 to $15^{\circ} \mathrm{C}[1,2]$. However, the characteristics of the battery when operating in such temperature range slightly below the optimum (Figure 4). Operation at lower temperatures may result in the evolution of hydrogen at high discharge currents. Increased operating temperature causes an increase in the rates of reactions, including degradation.

Batteries of different electrochemical systems have different resource. This phenomenon is associated with differences in the nature and intensity of occurrence of degradation processes.

In the nickel-cadmium batteries for operation are taken peace various degradation processes, the main of which are shown in Figure 5.
Rechargeable batteries which are operated in standby mode, must first provide power to the load during a specified time after its turning on. Thus, in the standby mode, the criterion of the ultimate condition of the battery is a bit capacity.

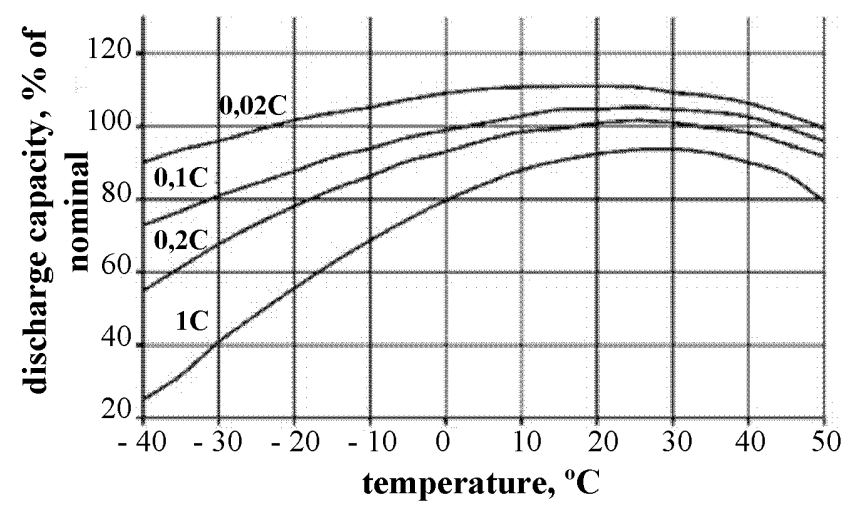

Figure 4. The dependence of discharge capacity of nickel-cadmium batteries from temperature discharge at various discharge currents

\begin{tabular}{|c|c|c|}
\hline \multicolumn{3}{|c|}{$\begin{array}{c}\text { The main processes causing degradation of the } \\
\text { characteristics of alkaline batteries }\end{array}$} \\
\hline$\downarrow$ & $\downarrow$ & $\downarrow$ \\
\hline $\begin{array}{l}\text { Occurring in } \\
\text { the electrolyte: } \\
\text { - carbonization } \\
\text { - reduction } \\
\text { - presence of } \\
\text { contaminants }\end{array}$ & $\begin{array}{l}\text { Occurring in the } \\
\text { construction: } \\
\text { - corrosion of } \\
\text { casing and } \\
\text { terminals } \\
\text { - bulging and } \\
\text { depressurization }\end{array}$ & $\begin{array}{l}\text { Occurring in } \\
\text { the electrodes: } \\
\text { - mechanical losses } \\
\text { of the active mass } \\
\text { - changes in } \\
\text { secondary structure } \\
\text { - crystallization }\end{array}$ \\
\hline \multicolumn{2}{|c|}{$\begin{array}{l}\text { Occurring in the separator: } \\
\text { - sprouting } \\
\text { - deformation and mechanical } \\
\text { destruction } \\
\text { - oxidation }\end{array}$} & $\begin{array}{l}\text { of the active mass } \\
\text { - oxidation of } \\
\text { graphite } \\
\text { - formation of } \\
\text { intermetallic }\end{array}$ \\
\hline
\end{tabular}

Figure 5. Degradation processes that reduce resource electrochemical batteries

In standby mode without a recharge batteries when they are operating the most important thing is keeping power. Review of the literature on this subject[1-3] and our own experimental studies have shown that the dominant degradation process in this case is the self-discharge. However, if you do not follow the operating instructions and the presence of defects in the protective case may occur corrosion housing batteries. In some cases, corrosion can lead to seal failure and battery leakage.

In standby mode, with sub-munitions (periodic or continuous) is characterized primarily grain growth of the active mass and the "memory effect". These processes cause a decrease in discharge capacity by reducing the efficiency of the volume of active mass. Other processes have significantly less impact on the willingness to give battery energy stored by the payload at the time of its inclusion. In some cases, loss of capacity may be caused by mechanical destruction of the electrodes. 
Studies of influence on the effect of various factors on the nature and intensity of degradation processes allowed the authors to develop a method for determining battery life, the principle of which is shown in Figure 6.

\section{Study of features of the normal operation mode} $\downarrow$

2. Defining characteristics of degradation processes

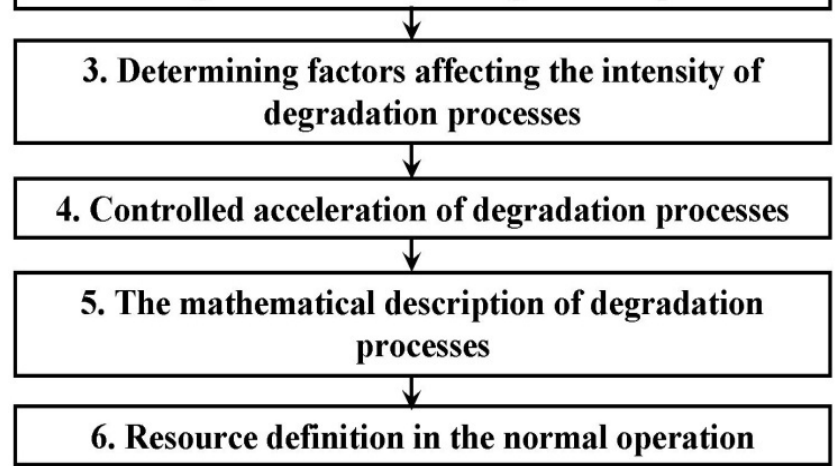

Figure 6. The scheme of the method for determining battery life, based on the physical accelerating degradation of the characteristics battery

Degradation processes occurring in batteries with different modes of operation (Figure 6, paragraph 1) have the characteristic features associated with the specifics of these modes. As a rule, there is no need to consider the totality of degradation processes, i.e. useful to distinguish the most probable processes leading to the battery limit state (Figure 6, paragraph 2). It is also inappropriate to consider all the factors influencing the nature and intensity of these processes. This is because the influence of some of them can be neglected due to the fact that the effect of their impact will be noticeable after reaching the ultimate state of the battery. Isolation of the most intense acting factors (Figure 6, paragraph 3) allows us to establish conditions under which the battery reaches the limit state much faster (Figure 6, paragraph 4), which in turn allows to determine quickly their resource (Figure 6, paragraph 6). If you exclude relevant factors you should be careful, because the error at this stage will lead to a possible change in the mechanism of degradation, significantly affect the reliability of the results. Study of the mechanism of degradation is also necessary to develop a mathematical description of the degradation characteristics depending on the intensity of the external effects (Figure 6, paragraph 5), which allows to determine the battery life under normal operation allows to defer in the results of tests in more aggressive conditions.

\section{The Influence of Self-Discharge of Electrochemical Batteries for Life, Operating in Standby Mode}

Self-discharge is the phenomenon of the spontaneous loss capacity for the open-circuit load. It is one of the benchmarks regular maintenance batteries aerospace applications $[1,2]$. In fact, the self-discharge rate determines the bit capacity of the battery in its work in stand-by or storage in a charged state. Thus, the current self-discharge is a key parameter in determining the reliability of the battery running in standby mode.

One of the factors causing self-discharge is often the closure of the external circuit due to the formation of conductive bridges in case the battery or battery between the battery. Such leaks can be avoided by adhering to the operational documentation.

The main reason for the phenomenon of self-discharge batteries is the internal processes that can only be predicted and taken into account or, at best, slow down.

The prerequisites for self-discharge are the following internal processes[3,4]:

- physic-chemical processes at the electrodes;

- the formation of bridges with the conductivity of the first kind in the material of the separator;

- shuttle redox reactions in the electrolyte.

The following main areas of potential test batteries to self discharge:

1. tested in real time;

2. mathematical modeling of self-discharge batteries on the basis of experimental data;

3. analog-to-physical modeling;

4. accelerated testing.

Duration of test batteries self discharge at a real-time unreasonably high. At the same time this method is widely known and permit to give the most accurate and adequate data on the possible self-discharge.

The use of mathematical modeling let to have a forecast of the self-discharge with less time. The accuracy and adequacy of the prediction by using a mathematical model may be different depending on the type of mathematical model and its implementation, but at any case the results of this experiment will be less reliable in comparison with the first of these methods.

To use for forecasting, analog physical modeling is promising. Unfortunately, the physical analogy that meets the requirements is not to have to known authors.

The concept of accelerated testing suggests the maximum reduction in the duration of works on the definition of given sizes while maintaining high accuracy and reliability of results.

Based on the test features characteristics of electrochemical batteries to self discharge, there are two main areas of research opportunities to reduce the overall duration of process:

1. acceleration of self-discharge;

2. shortening of diagnosis.

The most effective is to follow the first approach, as a characteristic time scale of the process of self-discharge is months and years. However, the successful solution of the self-discharge speed up the process and reducing its characteristic scale to days, and preferably also take steps to reduce the duration of diagnostic operations. 


\section{Theoretical Background of Thermal Aging Batteries}

The most promising is an accelerated self-discharge tests by accelerating the natural processes of self-discharge $\mathrm{Ni}-\mathrm{Cd}$ batteries.

Based on the mechanism of self-discharge, to accelerate the processes of the testing should be conducted at elevated temperatures.

Loss of nickel-cadmium of capacity batteries can be connected with the temperature and storage time equation (1), which is a special case of equation Erofeev[3,4]:

$$
\lg \mathrm{Q}_{\mathrm{p}}=\lg \mathrm{K}+\mathrm{n} \cdot \lg \tau,
$$

where $Q_{\mathrm{p}}$ - loss of capacity over time $\tau$.

$\lg K$ as a function of temperature can be determined from the Arrhenius equation:

$$
\lg \mathrm{K}=\mathrm{A}-\mathrm{b} / \mathrm{T}
$$

were $A$ and $b$ constants, experimentally determined for each battery.

To determine the constants of equations (1-2) for specific batteries it is necessary to conduct an experiment that includes an input control, thermal aging and output control, or only thermal aging of the indirect determination of residual capacity in real time. To determine the coefficients, the experiment should be repeated for temperatures of 50, 60, 70 and $80^{\circ} \mathrm{C}$.

Equation (1) at a fixed temperature is linear, thus, to extrapolate the loss of capacity for the desired time interval are enough input and output controls.

The data received as a result of this experiment is enough to assess the potential of self-discharge batteries in storage or operation in the standby mode.

\section{Determination of Loss Battery Capacity in the Process of Accelerated Tests}

The use of indirect methods for determining the capacity also reduces the duration of the tests.

The following methods to determine the capacity of:

1. The experimental method;

2. Experimental and computational method;

3. The analytical method.

The experimental method is in determining the capacity of the battery through a battery discharge, the constant current. The discharge capacity of the battery in this case is determined by the formula (3):

$$
Q_{d}=\int_{0}^{t_{d}} i_{d} d t
$$

where $Q_{\mathrm{d}}$ - bit capacity; $i_{\mathrm{d}}$ - discharge current, $\mathrm{t}_{\mathrm{d}}$ - time of discharge.

The main advantages of the method are high accuracy and simplicity. The method is widely used in engineering and is supported by regulatory documents[1,2]. However, the du- ration of the diagnostic battery using this method makes it unsuitable for using in accelerated testing.

Experimental and computational method involves the use to determine the capacity of (4) the discharge characteristics of batteries[4].

$$
\begin{aligned}
& \mathrm{U}=\mathrm{E}_{0}+\frac{\psi_{0}}{\mathrm{Q}_{\psi \mathrm{H}}}\left(\mathrm{Q}_{\psi \mathrm{H}}-\mathrm{I} \cdot \mathrm{t}\right)+\phi_{0} \exp \left(-3 \mathrm{I} \cdot \mathrm{t} / \mathrm{Q}_{\phi}\right)- \\
& -\mathrm{U}_{\mathrm{r} 0}\left\{1+\beta\left[1-\exp \left(-\alpha \cdot \mathrm{I}_{\mathrm{C}} / \mathrm{Q}_{\psi}-\mathrm{I} \cdot \mathrm{t}\right)\right]\right\},
\end{aligned}
$$

where $E_{0}$ - emf discharged battery, $\alpha, \beta$ - constant correction factors for nickel-cadmium batteries, $\alpha=0,26$ and $\beta=30$ [4]; $\psi$ - the difference between the emf corresponding to the point of intersection of the linear section of the curve $E=f(Q)$ with the $\mathrm{Y}$ axis, and the emf discharged battery; $Q_{\psi}$ - bit capacity of the battery in the 100-hour mode of discharge; $Q_{\psi \mathrm{H}}$ - discharge capacity of the battery in the 100-hour mode of discharge to final voltage; $\varphi_{0}$ - the difference between the voltage levels on the vertical axis, one of which corresponds to the beginning of the discharge curve, and another - at the intersection of the linear section with the ordinate axis; $Q_{\varphi}$ bit capacity of the battery when which the initial discharge curves of curvilinear portions are mated with straight, $U_{\mathrm{ro}}$ the battery voltage under load.

To determine the discharge capacity of the battery which is based on the obtained with the help of the method discharge curves you should use the formula (3).

Experimental and computational method allows you to indirectly determine the battery capacity on the basis of measurements without a discharge, which can significantly reduce the duration of the tests. However, to determine in real time the residual capacity of the battery self-discharge when this method is of little use, since it requires measuring the battery voltage under load, which creates additional capacity and leakage and requires to interrupt the process of thermal aging.

With the help of the analytical method can also determine the discharge capacity without a discharge, but in contrast to the experimental-calculation method, analytical method allows to obtain capacity directly and does not require additional calculations by formula (3).

An example of such relationship can serve as formula (5) [4]:

$$
\mathrm{Q}_{\mathrm{I} 0}=\frac{\alpha \cdot \mathrm{I}_{\mathrm{C}}}{\ln \beta \cdot \mathrm{U}_{\mathrm{r} 0}-\ln \left(\beta \cdot \mathrm{U}_{\mathrm{r} 0}+\mathrm{U}-\mathrm{E}\right)},
$$

where $Q_{\mathrm{I} 0}$ bit capacity, calculated using the measurements at constant current $I_{\mathrm{C}} ; \alpha, \beta$ - constant correction factors for nickel-cadmium batteries, $\alpha=0,26$ and $\beta=30[4] ; U_{\mathrm{r} 0}$ voltage drop across the internal resistance of charged battery in the discharge current 0.01 units of capacity, $U_{\mathrm{r} 0}=0.04 \mathrm{~V}[1]$; $U$ battery voltage, $E$ - emf the battery.

The accuracy of this of calculation method is the higher the larger the discharge current. Is to describe the processes of self-discharge, i.e. at low currents (3) is wrong.

The use of an analytical method for determining residual capacity during thermal aging is the most promising. This is 
explained by the fact that the experimental and calculated experimental methods require the discharge to the external load, and the analytical method allows only passive measurements.

To determine the loss of battery capacity for self- discharge, the authors, based on the equation of the discharge was derived the formula (6) describing the dependence of the loss of capacity from the current open circuit voltage and the battery time from the beginning of the experiment:

$$
\mathrm{Q}(\tau)=\mathrm{Q}_{0}\left(1-\exp \left[\frac{1}{\mathrm{r} \cdot \mathrm{Q}_{0}} \int_{0}^{\tau} \mathrm{U}(\mathrm{t}) \mathrm{dt}-\frac{\mathrm{U}_{0}}{\mathrm{r} \cdot \mathrm{Q}_{0}} \tau\right]\right),
$$

where $Q$ - loss of capacity; $Q_{0}$ - the initial discharge capacity; $U_{0}$ - initial stress; $r$ - active battery internal resistance; $\tau$ - the time of measurement relative to the start of the experiment; $U(t)$ - current open-circuit voltage battery.

Accordingly, the current residual battery capacity in the process of self-discharge can be defined as:

$$
\mathrm{Q}_{\text {res }}(\tau)=\mathrm{Q}_{0}-\mathrm{Q}(\tau) \text {, }
$$

where $Q_{\text {res }}$ - the residual battery capacity.

Analytical method for determining the capacity of using (6.7) allows to determine the residual battery capacity without interrupting the process of thermal aging, thereby significantly reducing the duration of the accelerated tests.

\section{Conclusions}

Use of a battery in standby mode without a recharge is typical for standby power systems.

During operation in the standby mode without recharging the battery is at condition of rest. External leaks and exposures, as a rule, are small and battery life define internal processes, such as self-discharge and a slow degradation of characteristics.

Self-discharge is one of the benchmarks regular maintenance aerospace batteries. In fact, the self-discharge rate determines the bit capacity of the battery in its work in stand-by or storage in a charged state. Thus, the intensity of self-discharge is a key parameter in determining battery life, running in standby mode.

Developed the concept of accelerated tests on the battery self-discharge can be used to predict the possible resource of nickel-cadmium batteries in storage or operating in standby mode, which is an important task.

Acceleration of degradation was carried out by exploiting the battery at elevated temperatures $\left(40-70^{\circ} \mathrm{C}\right)$. The method was tested on batteries 5NKP-6 and 6NKG-160, after which it was applied to determine the remaining battery life 27NKP-90 after prolonged use[3]. The use of accelerated aging at elevated temperatures it possible to achieve speedup degradation within 50-600. Thus, the developed method allows to quickly estimate the lifespan of the batteries and the possibility of further operation with high precision.

\section{REFERENCES}

[1] J. E. Gindelis, Electrochemical power source. Saratov, USSR: Press of Saratov university, 1984.

[2] S. V. Shirinsky, Theoretical aspects of accelerated tests on self-discharge of the alkaline batteries, Aerospace Engineering and Technology, 2(28), 37-40.

[3] S. V. Shirinsky, K. V. Bezruchko, Application of accelerated testing to assess the residual life of batteries, Bulletin of Dnipropetrovsk University, 9/2, 181-185.

[4] V. V. Romanov, J. M. Hashev, Electrochemical power source, $2^{\text {nd }}$ ed. Moscow, USSR: Soviet radio, 1978.

[5] A. A. Kharchenko, Analysis of the causes of degradation of nickel-cadmium batteries in the power plants of aircraft, Aerospace Engineering and Technology, 30, 266-272.

[6] Standard R IEC 60623-2002, Alkaline cells and batteries. Nickel-cadmium batteries, prismatic open. 01.07.2003, Moscow: Publishing standards, 2002.

[7] Standard R IEC 60622-2002, Alkaline cells and batteries. Nickel-cadmium batteries, sealed prismatic. 01.07.2003, Moscow: Publishing standards, 2002. 